\title{
Dynamic Monitoring of Key-Performance Indicators in Industrial Environments
}

\author{
Arnaldo Pereira*, Pierluigi Petrali ${ }^{\dagger}$, Arnaldo Pagani ${ }^{\dagger}$, José Barbosa*‡, Paulo Leitão*§ \\ * Polytechnic Institute of Bragança, Campus Sta Apolónia, 5300-253 Bragança, Portugal, \\ Email: \{arnaldo, pleitao, jbarbosa\}@ipb.pt \\ $\dagger$ Whirlpool Europe, Cassinetta di Biandronno, Italy, \\ Email: \{Pierluigi_Petrali, Arnaldo_Pagani\}@whirlpool.com \\ $\ddagger$ INESC-TEC, Rua Dr. Roberto Frias, 4200-465 Porto, Portugal \\ $\S$ LIACC - Artificial Intelligence and Computer Science Laboratory, Rua Campo Alegre 1021, 4169-007 Porto, Portugal
}

\begin{abstract}
Manufacturing companies generate huge amounts of relevant data that, potentially, can help the decision makers to monitor and supervise the health status of the shop-floor. Many problems are present that prevent this monitoring, namely the identification of which data should be collected, how to execute this collection or how this data should be analysed and displayed to the user. This paper deals with the last issue, where a dynamic and responsive User Interface is developed enabling the decision maker, having an in-the-mesh intervention, to constantly monitor relevant Key-Performance Indicators (KPI). The paper describes the development of such tool, considering a webbased approach under a platform agnostic perspective. The user interface presents an dynamic view over the aggregated KPI, where data is annotated with relevant warning information.
\end{abstract}

\section{INTRODUCTION}

Manufacturing companies are constantly faced with disturbance-related events [1]. These events, either with an internal or external source, are often unpredictable and have a negative impact in the normal operating situation of the company creating a performance degradation. Several impacts are directly related with this performance degradation leading companies to delay deliveries, pay penalties to costumers and see their brand confidence degraded.

To this extent, the company decision makers must be equipped with the necessary tools for a sustained and prompt decision making process, particularly those taken at the tactical and strategic level [2]. Decision makers rely on several Key Performance Indicators (KPIs) to assess the status of the company current situation. KPIs such as Lead Time, Takt Time and Overall Equipment Effectiveness (OEE) are used as verification metrics. Of major importance for the proper development of an effective KPI monitoring tool is the data collection, its aggregation and easy display in a multi-platform environment, making such tool a real added-value asset.

The aforementioned topic is being currently greatly addressed in many research and development works worldwide, alongside with several other topics is diverse domains of knowledge [3], [4], under the umbrella of the $4^{\text {th }}$ industrial revolution. This is pushing the current state-of-the-art forward, empowering companies with the necessary set of tools to better face these ever demanding requirements.
Several national and international financing programs have been or are being deployed, namely the Industrie 4.0 [5] in Germany, "La Nouvelle France Industrielle" in France, "Smart Industry" in Netherlands, "Industria Conectada 4.0" in Spain, "Innovate UK" in United Kingdom and "Fabbrica Intelligente" in Italy, the "Industrial Internet" initiative in the United States [6] and the "Made in China 2025" program in China, creating the appropriate stimulus for this revolution. Despite of the different funding programs and research groups, a common research denominator can be found, mainly focusing the digitization, modularization, seamless communication, reconfiguration and self-* topics.

The PERFoRM (Production harmonizEd Reconfiguration of Flexible Robots and Machinery) [7] project is developed considering the easy system reconfiguration of robots and machinery, promoting also a seamless integration of legacy and newly developed solutions. The easy connection and a transparent data exchange between the different hardware and software components and/or modules of different data sources is also ensured by the use of a common data model. Higher level tools, particularly those found at higher levels of the ISA-95 pyramid are also envisioned, focusing particularly scheduling, simulation and visualization.

This paper focuses on the development of an application for the dynamic and on-line monitoring of KPIs in industrial environments, applied particularly to the production of microwave appliances, allowing the correlation of different Key Business Factors (KBFs). This application allows the decision makers to be equipped with the necessary tool to, dynamically and in a real time manner, monitor the relevant KPIs that influence the performance of the manufacturing system, allowing them to promptly act and correct possible trending situations. The proposed solution was deployed using real collected data, being possible to test and validate the designed solution.

The rest of the paper is organized as follows: Section II overviews the PERFoRM concept regarding the seamless reconfiguration of robotics and machinery, and Section III introduces the microwave production system use case. Section IV describes the architecture of the dynamic KPI monitoring tool and Section V presents the deployment of this solution for the industrial use case. Finally, Section VI rounds up the 
paper with the conclusions and points out the future work.

\section{PERFoRM CONCEPT TO THE SEAMLESS RECONFIGURATION}

The PERFoRM system architecture [7], depicted in Figure 1 presents a modular concept to implement the industrial needs for the current industrial revolution. PERFoRM allows the transparent and interoperable communication between different hardware and software components at all the ISA95 levels. At the hardware level, i.e. at lower levels of the ISA-95 pyramid, devices such as PLCs (Programmable Logic Controllers) and industrial robots are able to be connected. Also, components at higher ISA-95 levels, such as MES (Manufacturing Execution Systems), scheduling, data analytics, simulation or visualization tools are also capable to be plugged in a PERFoRM system.

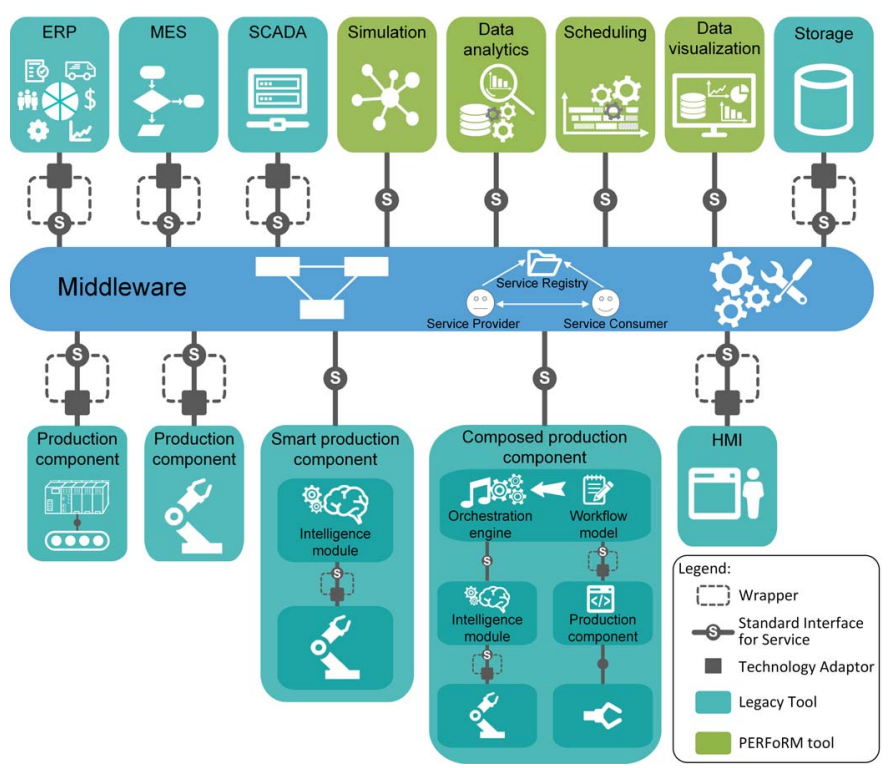

Figure 1. PERFoRM system architecture.

This level-agnostic pluggability enables a seamless production system reconfiguration, where heterogeneous hardware and software production components, addressing the different ISA-95 levels, expose their functionalities as services. An industrial middleware acts as a distributed service-based integration layer supporting the transparent interoperability of these individual components.

Due to the heterogeneity nature of the companies' components, a common data model was adopted, based on AutomationML, acting as the data exchange format, shared between the PERFoRM-compliant architectural elements, covering the semantic needs associated to each entity [8]. Additionally, the definition of standard interfaces assumes a crucial role in the architectural design issue addressing the seamless interoperability feature in real industrial environments. These interfaces, expose and describe services in an unique, standardized and transparent way, enhancing the seamless interoperability and pluggability.
Legacy devices and data sources, not following the PERFoRM specifications, need to be integrated by exposing their data in a "PERFoRM compliant" manner. Therefore, technology adapters are key elements to connect these legacy systems to the PERFoRM middleware, converting the legacy data into the PERFoRM's data model. The tools developed according to the PERFoRM data model don't need the use of technological adapters (as observed in Figure 1).

Finally, PERFoRM adopts the human as a flexibility driver and considering two major roles. First, the human can be considered as "human-in-the-loop" where he can oversee and control a physical process [9]. Secondly, in a "human-in-themesh" situation, the human can receive alerts, analyse and change planning scenarios. Finally, the human role can have a Janus effect where it can play both roles simultaneously.

\section{Microwave Oven Production System}

Whirlpool is a world leader in large domestic appliances with a strongly presence in Europe with more than 20 factories in 14 sites and development centres located in 6 countries. In particular, the Whirlpool Microwave factory at Biandronno (Italy) site produces up to 450.000 built-in microwave ovens per year.

The production process is composed of several steps (see Figure 2): material incoming, cavity line fabrication (to produce the internal cavity of the oven), door fabrication and assembly, part fabrication and silk screening, final assembly (of cavity, door and all other components), testing, repairing bay and packaging. From the logical point of view, the factory is organized according to the Whirlpool Production System: the material and information flows are described through the Value Stream Map, which is highlighting the relationship between production blocks in terms of complexity, buffers, sequence, timing and resources.

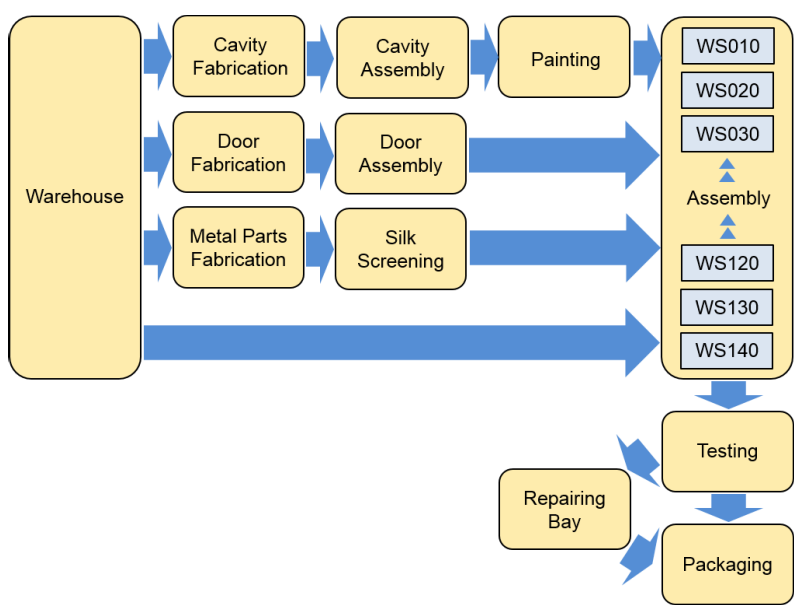

Figure 2. Microwave factory block layout.

In order to simplify and standardize the way in which the factory is described, the main static parameters that are considered critical for their influence on the business results have been grouped and codified under the concept of Key 
Business Factors (KBF). Each time a factory is modified, in a permanent or semi-permanent way, one or more KBF are changed consequently.

Within the PERFoRM project, the main objective of the Whirlpool use case is to establish a real time monitoring system empowering the decision maker user with a tool for a continuous supervision of KPIs, using appropriate user interfaces (UI). Besides the responsive feature, this tool should be able to warn and alert the user for critical situations, namely those that at a bare eye would be difficult to oversee. This tool should also correlate crucial KPIs and KBFs, increasing the system reconfiguration responsiveness due to a faster assessment of the KPI-KBF interdependencies. Figure 3 shows the model of the latest that was implemented within PERFoRM.

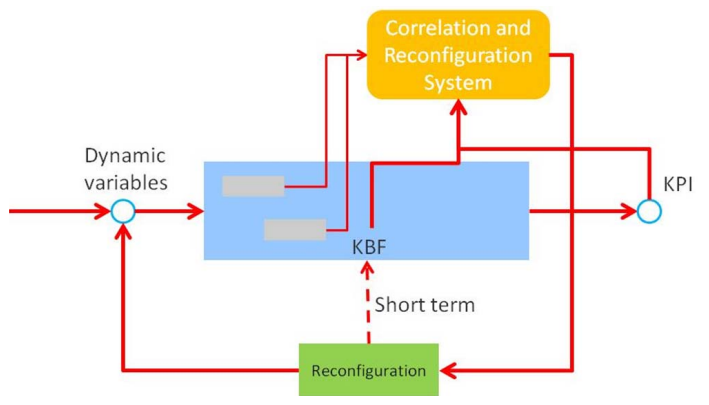

Figure 3. Reconfiguration system model.

The model objectives consist, essentially, in a particular and dynamic system which is able to manage the following actions: recognize the input, identify the $\mathrm{KBF}$, define a relevant model, evaluate the output (i.e. the KPI value), compare inputs (KBFs and KPIs in order to choose the actions needed to obtain the system reconfiguration and to improve the output), apply a sensitivity analysis aiming to define the relationships (i.e. sensitivity factors) among KBFs and KPIs, and validate the model consistency.

Such a tool assumes a critical importance if one considers that currently the KPI identification system is only able to collect equipment information with a high granularity level, particularly regarding their batch size, production activity, resource consumption, quality data and operative status. Thanks to this gathered data, the system can dynamically point out both KPI (e.g., Output, Lead Time, Takt Time and OEE) and KBF (e.g., Worked Hours/year and Total Demand) separately. Therefore, the tool in this paper presents a solution to act as an information aggregation so that it can be immediately useful, in a automatic and flexible way, offering the ability to detect, earlier as possible, disturbances or performance degradation.

\section{Dynamic KPI Monitoring TOOL}

The KPI monitoring tool is a solution to support decisionmaking strategies, by monitoring KPIs, detecting trends and deviations, and performing what-if game based on the variation of KBFs and generating the associated KPI implication.

Architecturally, the tool is composed of the KPI Calculation \& Statistical Quality Control Module, What-if-game Module,
Data Service Module, Data Handler Module and Visualization Module, as depicted in Figure 4.

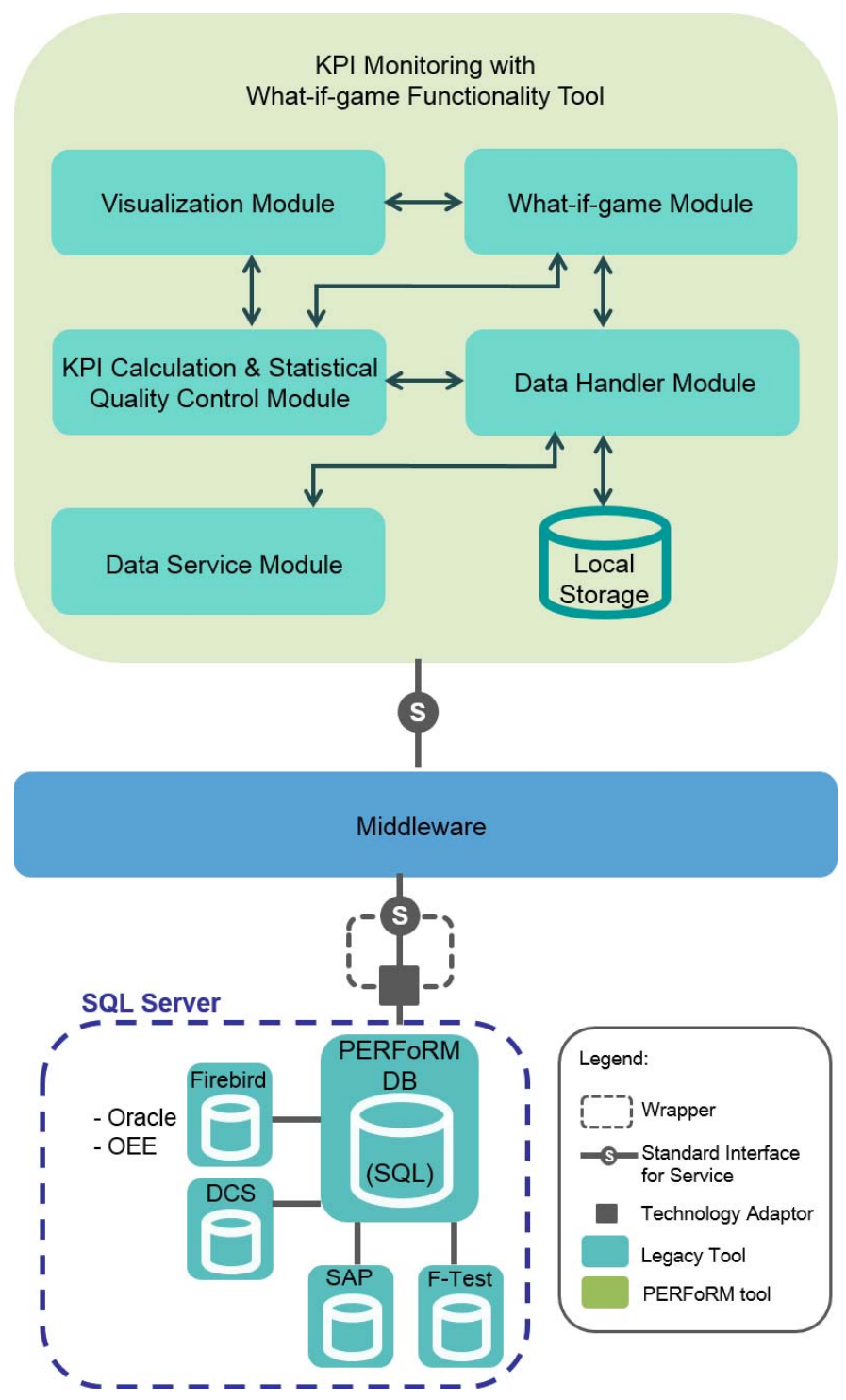

Figure 4. Architecture of the Dynamic and On-line KPI-monitoring Tool.

These modules are described in the following sub-sections.

\section{A. KPI Calculation \& Statistical Quality Control Module}

The KPI Calculation \& Statistical Quality Control Module is responsible for the calculation of the KPIs, as defined and evaluated in [10]. For this purpose, the set of KBFs presented in Table I are used as input while two different sets of KPIs are calculated allowing to assess the evolution of the undergoing industrial process. The first set, presented in Table II (with the respective mathematical formulation), is composed of KPIs related with the $M$ monitored working stations. On the other hand, the second set of KPIs refers to the overall system parameters (see Table III).

Used by many industrial sectors, the Six-Sigma is a set of techniques and tools for process improvement [11], targeting 
Table I

KEY BUSINESS FACTORS

\begin{tabular}{|c|l|c|}
\hline Symbol & \multicolumn{1}{|c|}{ Description } & Unit \\
\hline$W d$ & Number of working day during the year & day (d) \\
\hline$h_{S}$ & Hour for each shift & hour (h) \\
\hline$S_{d}$ & Number of shift for each working day & number \\
\hline$T D$ & $\begin{array}{l}\text { Total Demand: number of pcs requested for } \\
\text { each hour }\end{array}$ & pcs/h \\
\hline$C T$ & $\begin{array}{l}\text { Cycle Time: time between the beginning } \\
\text { and end of the process of making a product }\end{array}$ & second (s) \\
\hline$O M$ & $\begin{array}{l}\text { The number of operator/machine that } \\
\text { are involved in the same working station }\end{array}$ & number \\
\hline$N C$ & $\begin{array}{l}\text { Non-conforming product } \\
\text { Availability: percentage of production } \\
\text { losses due to availability problem }\end{array}$ & $\begin{array}{l}\text { Performance: percentage of production losses } \\
\text { due to performance problem }\end{array}$ \\
\hline$P T$ & $\begin{array}{l}\text { Set Up Time: the time required to set up a } \\
\text { device for production of a new batch }\end{array}$ & $\mathrm{s} / \mathrm{batch}$ \\
\hline$P$
\end{tabular}

Table II

KPIS FOR EACH WORKING STATION (CALCULATION FORMULAS)

\begin{tabular}{|l|c|}
\hline \multicolumn{1}{|c|}{ KPI } & Unit \\
\hline Total Cycle Time $($ TCT) $=C T * O M$ & $\mathrm{s}$ \\
\hline $\begin{array}{l}\text { Actual Daily Machine Availability }(A D M A)= \\
h_{S} * 60 * 60 * S_{d} * O M * \text { Av }\end{array}$ & $\mathrm{s} / \mathrm{d}$ \\
\hline $\begin{array}{l}\text { Actual Processing Time }(\text { APT })= \\
\text { TCT }+ \text { ST } *(1+N C)\end{array}$ & $\mathrm{s} / \mathrm{pcs}$ \\
\hline Output $=$ Throughput $=$ ADMA $* A P T * P$ & $\mathrm{pcs} / \mathrm{d}$ \\
\hline LeadTime $($ LT) $=(1 /$ Output $) * 3600$ & $\mathrm{s} / \mathrm{pcs}$ \\
\hline $\begin{array}{l}\text { Takt Time }(T T)= \\
\left(h_{S} * 60 * 60 * S_{d} * W d\right) /\left(T D * h_{S} * W d\right)\end{array}$ & $\mathrm{s}$ \\
\hline $\begin{array}{l}\text { Overall Equipment Ef fectiveness }(O E E)= \\
(1-N C) * A_{v} * P\end{array}$ & $\%$ \\
\hline $\begin{array}{l}\text { Time of Stock } \\
\left.\text { 3600/LT } T_{i+1}\right) * \text { LT }_{i+1}, i=1, \ldots, M-1\end{array}$ & $\mathrm{~s} / \mathrm{pcs}$ \\
\hline
\end{tabular}

the variability reduction in key product quality indicators, which goal is the achievement of a very low level of defects. Assuming that the improvement of process performance and a reduced variability of the key parameters of the running manufacturing processes are critical features, the module is responsible for the statistical quality control of the temporal evolution of the KPIs, to ensure the expected quality of the appliances (microwave ovens) in production.

To perform the on-line statistical process control, a control chart for each KPI, relative to each of the stations, graphically

Table III

KPIS FOR THE OVERALL SYSTEM (CALCULATION FORMULAS)

\begin{tabular}{|l|c|}
\hline \multicolumn{1}{|c|}{ KPI } & Unit \\
\hline Bottleneck Machine $=\max \left\{L T_{m}: m=1,2, \ldots, M\right\}$ & $\mathrm{s} / \mathrm{pcs}$ \\
\hline Output/Hour $\left(O_{h}\right)=3600 /$ Bottleneck & $\mathrm{pcs} / \mathrm{h}$ \\
\hline Theoretical Production Time $=\sum_{m=1}^{M}$ TCT & $\mathrm{s}$ \\
\hline Total Production Time $=\sum_{m=1}^{M}\left(L T_{m} * O M_{m}\right)$ & $\mathrm{s}$ \\
\hline $\begin{array}{l}\text { Total Actual Lead Time }=\sum_{m=1}^{M}\left(L T_{m} * O M_{m}\right) \\
+\sum_{m=1}^{M} \text { Time of Stock }\end{array}$ & $\mathrm{s}$ \\
\hline Stock $($ pcs $)=$ Batch Size $*$ Item & $\mathrm{pcs}$ \\
\hline Stock $($ hours $)=$ Stock $($ pcs $) / O_{h}$ & $\mathrm{pcs} / \mathrm{h}$ \\
\hline
\end{tabular}

displays the evolution of the KPI over time. The control chart plots the KPI's data points against some control lines. A centreline representing the average value of the KPI in question is displayed. Three other pairs of lines surround this centreline at one-sigma, two-sigma and three-sigma (i.e. at once, twice and three times the variance of the KPI). All these lines are used to assess some pattern denouncing out-of-control condition. Notably the Western Electric Rules [12] are used:

Rule 1: One data point falls outside the three-sigma control limits.

Rule 2: Two out of three consecutive data points fall beyond the two-sigma warning limits.

Rule 3: Four out of five consecutive data points fall beyond the one-sigma limit, on the same side of the centreline.

Rule 4: Eight consecutive data points fall on one side of the centre line.

Additionally, the following rules were also considered: [11]:

Rule 5: Six data points in a row steadily increasing or decreasing.

Rule 6: Fifteen data points in a row fall within the one-sigma limits (stratification).

Rule 7: Fourteen data points in a row alternating up and down.

Rule 8: Eight points in a row on both sides of the centreline with none falling within the one-sigma limits (mixture).

Points that present a pattern abnormality are signalled (e.g., be painted red) for better visualization. Additionally, the points that falls between the two-sigma and the three-sigma limits are stand out by the use of appropriate interval limit colours.

\section{B. What-if-game Module}

The what-if-game mode, provided by this module, adds an extra functionality by introducing degrees of freedom (DoF) intervals on the KBFs, allowing to foresee how the system behave within the specified intervals. Therefore, new production scenarios can be elaborated considering a more sustained decision-making process, based on the obtained expected KPIs. This mode is initiated by the decision maker for possible scenarios assessment or e.g., in order to mitigate the deviation of the actual KPI values from target values.

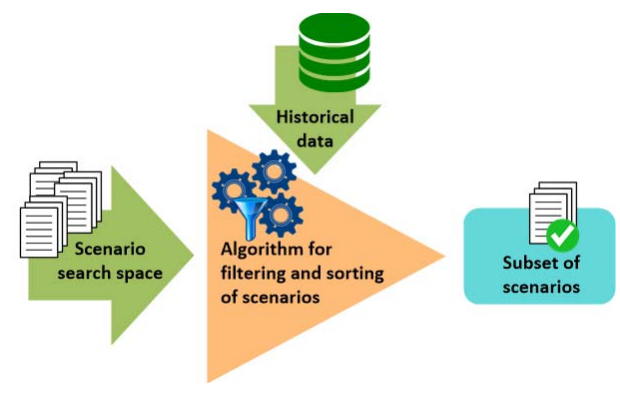

Figure 5. Scenario generation process.

The simultaneous interval variation of several KBFs increases the scenarios combinatorial search space. Therefore, 
and without compromising the tool responsiveness, an intelligent scenario generation is implemented, prioritizing the calculation of the most promising scenarios (as shown in Figure 5), in a similar way to that proposed in [2].

The What-if-game Module uses internal info and the KPI Calculation \& Statistical Quality Control Module to generate relevant outputs that must be provided to the Visualization Module, allowing the presentation of data in a way that could be evaluated at a glance.

\section{Data Service, Data Handler and Visualization modules}

To properly operate, the tool needs to access the production data, which in this case is available in a PERFoRM-compliant way, following the specified data model (as referred in section II), by means of the use of a technological adapter that converts the native data structure into the PERFoRM data model. Thus, from the point of view of the tool, the data access is transparent, executed by the Data Service Module, and operates on a request-reply manner, for a more static and historical data collection, or using a publish-subscribe mechanism, for a real-time data collection.

In addition to this remote data access, local data is required to support the continuous operation of the KPI Calculation \& Statistical Quality Control Module. Therefore, the Data Handler Module manages the local repository and evaluates whether there is the need to trigger further data acquisition via the Data Service Module.

Finally, the Visualization Module renders the information in a suitable way to be presented in web-based UI applications.

\section{IMPLEMENTATION AND DEPLOYMENT OF THE KPI-MONITORING TOOL}

The choice of technological solutions to implement the tool was guided by the option of having multi-device compatibility, web-based tools and the adoption of mature technologies. This kind of considerations leaded to the option of create a Javabased dynamic Web application, using the JavaServer Pages (JSP) technology [13]. To ensure the multi-device compatibility, a clean and responsive HTML5 [14] template for the front-end web development was used, namely the freehtml5.co "Cache: Free HTML5 Bootstrap Template". The developed application was deployed in an Apache Tomcat [15] server (other compatible web server with a servlet engine, like Jetty or GlassFish, can also be used).

\section{A. KPI monitoring mode}

The tool UI presents data following a production stations layout sorted order, displaying the several KPIs for each one of them (see Figure 6 - left). The target and the actual KPIs values are shown, as well as their evolution trend (positive or negative). A colour scheme enriches the visual experience, enabling the user to quickly detect problematic situations. Additionally, a control chart for each KPI is accessible by opening a new UI perspective, displaying its timed evolution, as shown in Figure 6 - right. This screenshot also signals the practical implementation of the previously described rule 1.
The implementation of the control charts uses the Highcharts 5.0.5 [16], which is a library written in JavaScript that allows the creation of dynamic charts.

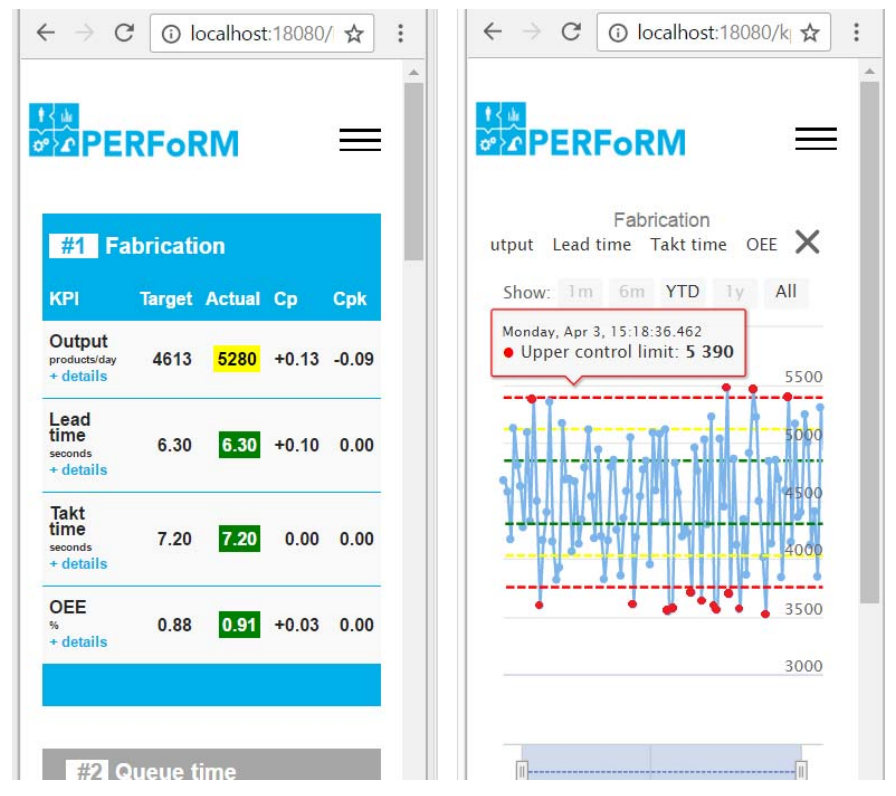

Figure 6. Two views of the tool in KPI Monitoring mode.

A request-reply mechanism, using RESTful Web Services, implements the collection of the historical data. The following code excerpt, depicts the used Java API for RESTful Web Services (JAX-RS) [17], focusing on the implementation of a getValue request to the industrial middleware.

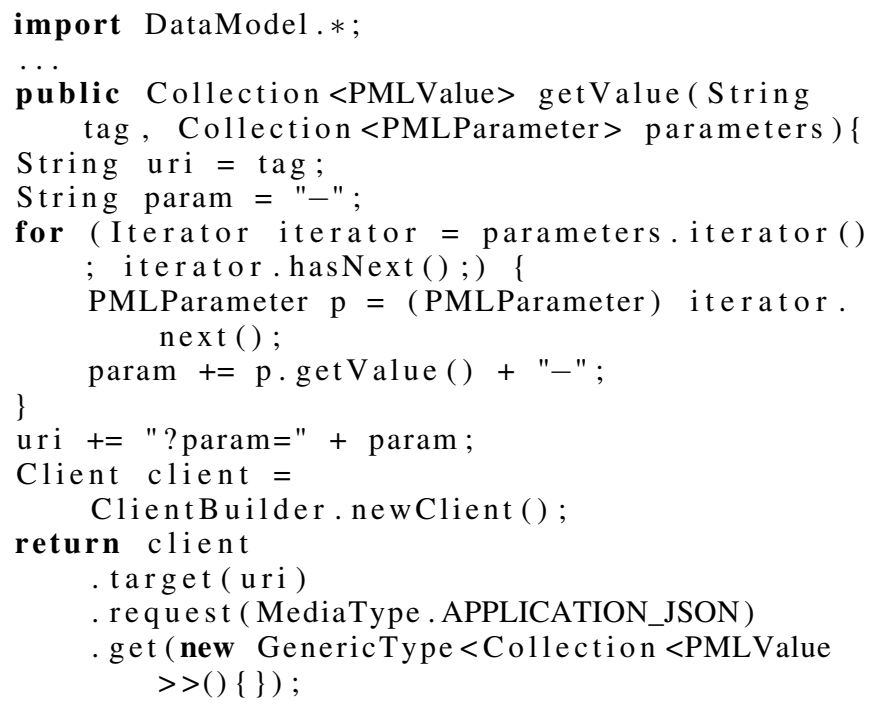

On the other hand, the on-line refresh of the data presented by the tool is achieved by using the publish-subscribe mechanism implemented using the Message Queuing Telemetry Transport (MQTT) protocol [18]. For this purpose, the Eclipse Paho client was used. The following excerpt depicts a topic subscription for a Quality of Service of 2.

import org.eclipse.paho. client.mqttv 3 .* ... 
MqttClient client $=$ new MqttClient $($ this serverURI, this. clientId);

MqttConnectOptions connOpts = new MqttConnectOptions ();

connOpts. setCleanSession ( false);

client. connect (connOpts); // connection to broker

$\operatorname{try}\{$

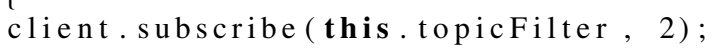

\section{B. What-if game mode}

The what-if-game mode functionality presents data in a spider diagram manner, aggregating all the relevant KPIs into one graphical display, see Figure 7. The figure left side shows the overall system what-if KPIs results while, on the right part of the figure, the user can study other levels of granularity by searching KPIs at processing/station level.

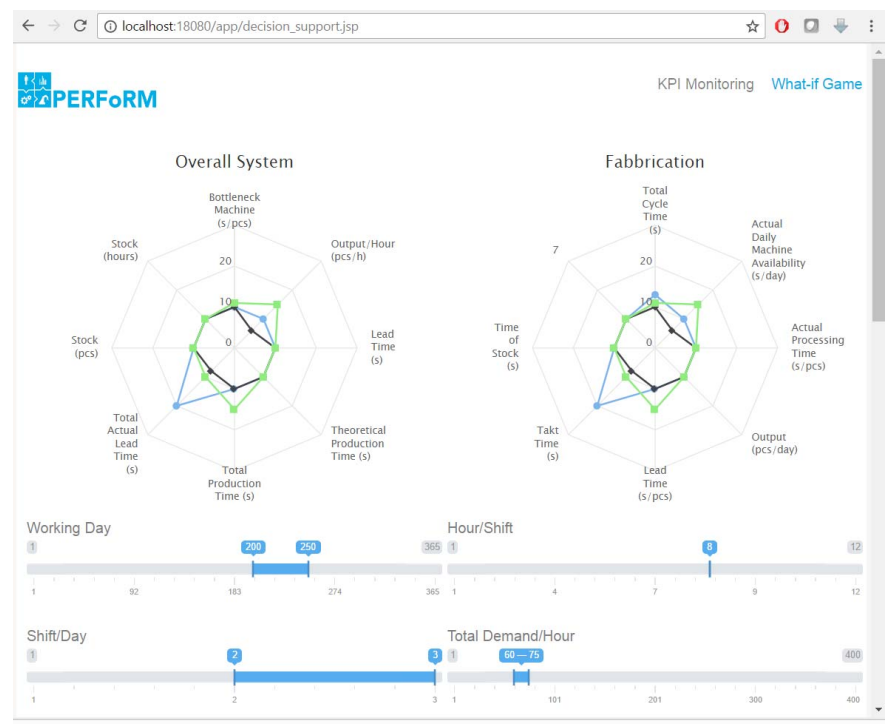

Figure 7. View of the tool in What-if Game mode.

The decision maker, can change the desired KBFs by adjusting the sliders located at the lower part. With this, a new set of solutions will be displayed to the user allowing a thorough impact assessment of these adaptations.

\section{CONCLUSION}

The vast amount of generated data at shop-floor has the potential, when properly analysed and monitored, to derive the system health situation allowing a responsive reaction to current or future problematic situations.

This paper presented a tool for the dynamic monitoring and visualization of KPIs in a microwave oven production line, which improves the decision process by empowering the user, considered in this case as human-in-the-mesh, with a valuable perspective of the crucial KPIs. Two distinct abstraction levels were considered: in the first, an aggregated KPI view is presented, allowing a fast overview of the shop-floor situation, while in the second, the decision maker is presented with a detailed KPI view, in a time line perspective.
The tool is enhanced with a what-if game functionality that enables the user with a playground to play with the DoF for the KBFs while observing their impact on the KPIs. This feature helps the user to, e.g., define the KBFs in a timely manner.

Future work will be dedicated to intensive testing at the factory, tackling the challenges related to the integration of the tool with the middleware and the configuration and use of the technological adapter connected to the production database.

\section{ACKNOWLEDGMENT}

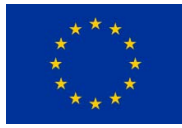

This project has received funding from the European Union's Horizon 2020 research and innovation programme under grant agreement No 680435

\section{REFERENCES}

[1] P. Leitão and J. Barbosa, "Disturbance Detection, Recover and Prediction in Holonic Manufacturing Control," in Proceedings of the IEEE Workshop on Distributed Intelligent Systems: Collective Intelligence and Its Applications, pp. 133-138, 2006.

[2] P. Leitão, N. Rodrigues, and J. Barbosa, "What-if game simulation in agent-based strategic production planners," in 20th Conference on Emerging Technologies \& Factory Automation (ETFA'15), 2015.

[3] K. Schwab, The Fourth Industrial Revolution. VINT research report, World Economic Forum, 2016.

[4] H. Bauer, C. Baur, G. Camplone, and et. al., "Industry 4.0: How to navigate digitization of the manufacturing sector," tech. rep., McKinsey Digital, 2015.

[5] H. Kagermann, W. Wahlster, and J. Helbig, "Securing the future of German manufacturing industry: Recommendations for implementing the strategic initiative INDUSTRIE 4.0," tech. rep., ACATECH - German National Academy of Science and Engineering, 2013.

[6] S.-W. Lin, B. Miller, J. Durand, R. Joshi, P. Didier, and A. C. et al., "Industrial Internet Reference Architecture," tech. rep., Industrial Internet Consortium (IIC), June 2015.

[7] P. Leitão, J. Barbosa, A. Pereira, J. Barata, and A. Colombo, "Specification of the PERFoRM architecture for seamless production system reconfiguration," in Proceedings of the $42^{\text {nd }}$ Annual Conference of IEEE Industrial Electronics Society (IECON'16), pp. 5729-5734, 2016.

[8] R. S. Peres, M. Parreira-Rocha, A. D. Rocha, J. Barbosa, P. Leitão, and J. Barata, "Selection of a data exchange format for industry 4.0 manufacturing systems," in 42nd Annual Conference of the IEEE Industrial Electronics Society (IECON'16), pp. 5723-5728, 2016.

[9] P. Fantini, G. Tavola, M. Taisch, J. Barbosa, P. Leitão, Y. Liu, M. S. Sayed, and N. Lohse, "Exploring the integration of the human as a flexibility factor in CPS enabled manufacturing environments: Methodology and results," in 42nd Annual Conference of the IEEE Industrial Electronics Society (IECON'16), pp. 5711-5716, 2016.

[10] F. Boschi, C. Zanetti, G. Tavola, and M. Taisch, "Functional Requirements for Reconfigurable and Flexible Cyber-Physical System ," in 42nd Annual Conference of the IEEE Industrial Electronics Society (IECON'16), pp. 5717-5722, 2016.

[11] D. C. Montgomery, Introduction to Statistical Quality Control. John Wiley \& Sons, Inc., 6th ed., 2009.

[12] W. Electric, Statistical Quality Control Handbook. Western Electric Corporation, 1956.

[13] L. Pekowsky, JavaServer Pages. Addison-Wesley, 2004.

[14] B. Frain, Responsive Web Design with HTML5 and CSS3. Packt Publishing, 2012.

[15] A. Vukotic and J. Goodwill, Apache Tomcat 7. Apress, 2011.

[16] J. Kuan, Learning Highcharts. Packt Publishing, 2012.

[17] S. Gulabani, Developing RESTful Web Services with Jersey 2.0. Packt Publishing, 2013.

[18] OASIS, "MQTT Version 3.1.1 Plus Errata 01." http://docs.oasis-open. org/mqtt/mqtt/v3.1.1/mqtt-v3.1.1.doc, December 2015 . 